\title{
The Topological Entropy of Iterated Piecewise Affine Maps is Uncomputable
}

\author{
Pascal Koiran \\ Laboratoire de l'Informatique du Parallélisme, Ecole Normale Supérieure de Lyon-CNRS, \\ 46, allée d'Italie, 69364 Lyon Cedex 07, France \\ e-mail: Pascal.Koiraneens-lyon.fr \\ http://wWw.ens-lyon.fr/ koiran
}

received Nov 29, 2000, accepted Dec 20, 2001.

\begin{abstract}
We show that it is impossible to compute (or even to approximate) the topological entropy of a continuous piecewise affine function in dimension four. The same result holds for saturated linear functions in unbounded dimension. We ask whether the topological entropy of a piecewise affine function is always a computable real number, and conversely whether every non-negative computable real number can be obtained as the topological entropy of a piecewise affine function. It seems that these two questions are also open for cellular automata.
\end{abstract}

Keywords: topological entropy, piecewise affine functions, saturated linear functions, cellular automata

\section{Introduction}

There is an active line of research in which dynamical systems are studied from an effective point of view. Here one main goal is to clarify the boundary between decidable and undecidable properties of dynamical systems. Cellular automata and iterated piecewise-affine maps are two classes of systems for which this endeavour has been particularly successful. The present paper is devoted to iterated piecewise-affine continuous maps. We show that it is undecidable whether the topological entropy of such a function defined on the fourdimensional cube $[-1,1]^{4}$ is equal to zero (precise definitions are given in section 2). A similar result was obtained several years ago for one-dimensional cellular automata [Z] by a reduction from the nilpotency problem, which was proved undecidable in [3]. We also use a reduction from the nilpotency problem, albeit of piecewise-affine maps instead of cellular automata. The undecidability of this problem was only established recently in [U]]. As in [2] it follows from the proof that the topological entropy cannot be computed, even approximately. We also give an undecidability result for saturated linear systems, which are a restricted class of iterated piecewise-affine maps. These systems have been of interest in control theory, see for instance [7]. Finally we show that there exists a dimension $n$ and nilpotent saturated linear maps $f: \mathbb{R}^{n} \rightarrow \mathbb{R}^{n}$ such that $f^{k}$ (the $k$-th iterate of $f$ ) is not identically zero, where $k$ is an arbitrarily large integer. This clarifies an observation of [T]. It is however still unknown whether there exists $n$ such that the nilpotency problem for saturated linear functions in dimension $n$ is undecidable.

We conclude this introduction with an open problem: is the topological entropy of a piecewise-affine map always a computable real number? The concept of computable real number is explained in many texts on recursive function theory or recursive analysis, see e.g. [4, 9]. Here as elsewhere we assume that the piecewise-affine map has only rational 
coefficients, see section 2.2. Our undecidability result does not seem to provide an answer to this question. It is however clear from the definition of topological entropy that this number is always in the arithmetical hierarchy of real numbers studied in [10]. Conversely, one can ask whether every computable real number can be obtained as the topological entropy of an iterated piecewise-affine map. These two questions also make sense for cellular automata, and it seems that they have not been addressed so far.

\section{Dynamical Systems}

We consider dynamical systems defined by iteration of a map $f: X \rightarrow X$. A trajectory of such a system is a sequence $\left(x_{t}\right)_{t \in \mathbb{N}}$ of points of $X$ such that $x_{t+1}=f\left(x_{t}\right)$ for all $t \in \mathbb{N}$. We are mainly interested in the case where $f$ is piecewise affine and $X$ is the $d$-dimensional cube $[-1,1]^{d}$.

\subsection{The Topological Entropy}

Let $(X, d)$ be a compact metric space and $f: X \rightarrow X$ an arbitrary (possibly discontinuous) map. From $d$ and $f$ we can construct a family $\left(d_{n}\right)$ of distance functions as follows: $d_{n}(x, y)=\max _{0 \leq i \leq n} d\left(f^{i}(x), f^{i}(y)\right)$. We say that a subset $F$ of $X$ is an $\varepsilon$-spanning set for $\left(X, d_{n}\right)$ if for all $x \in X$ there exists $y \in F$ such that $d_{n}(x, y) \leq \varepsilon$. There is a dual notion of $\varepsilon$-separated set: this is a subset $F$ of $X$ such $d_{n}(x, y)>\varepsilon$ for any distinct points $x$ and $y$ of $F$. We denote by $r_{n}(\varepsilon)$ the smallest cardinality of any $\varepsilon$-spanning set of $\left(X, d_{n}\right)$, and by $s_{n}(\varepsilon)$ the largest cardinality of any $\varepsilon$-separated set. We also write $r_{n}(\varepsilon, f)$ and $s_{n}(\varepsilon, f)$ when $f$ is not clear from the context. It follows from the compactness of $X$ that $r_{n}(\varepsilon)$ and $s_{n}(\varepsilon)$ are always finite.

One can show that the two limits

$$
\lim _{\varepsilon \rightarrow 0} \limsup _{n \rightarrow \infty} \ln r_{n}(\varepsilon) / n
$$

and

$$
\lim _{\varepsilon \rightarrow 0} \limsup _{n \rightarrow \infty} \ln s_{n}(\varepsilon) / n
$$

exist and are equal (they may be infinite). This common limit is called the topological entropy of $f$, and is denoted $h(f)$. For more details we refer the reader to [5] and [8] (the latter reference defines the topological entropy only for uniformly continuous maps).

Consider for instance the case where $f$ is a nilpotent map. This means that there exists an integer $m$ such that $f^{m}(X)=\{0\}$, where 0 is a distinguished point of $X$. Then $h(f)=0$ since $r_{n}(\varepsilon)$ is bounded independently from $n$ by $r_{m}(\varepsilon)$.

As a second example, consider the "tent map" $\phi:[0,1] \rightarrow[0,1]$ defined by: $\phi(x)=2 x$ for $x \in[0,1 / 2]$ and $\phi(x)=2(1-x)$ for $x \in[1 / 2,1]$. The $n$-th iterate of $\phi$ is made of $2^{n}$ "laps" (monotone pieces). This implies that $h(\phi)=\ln 2$ [6] (this can also be checked directly from the definition of topological entropy). Here as in the remainder of the paper, the topological entropy is computed with respect to the Euclidean distance. The example of the tent map will be used later. In fact we will only need to know that the topological entropy of $\phi$ is nonzero.

\subsection{Piecewise-affine maps}

A function $f: \mathbb{R}^{p} \rightarrow \mathbb{R}^{q}$ is piecewise affine if its graph is a semilinear subset of $\mathbb{R}^{p} \times \mathbb{R}^{q}$ (i.e., a boolean combination of halfspaces). We will assume that the corresponding halfspaces are defined by affine inequalities with rational coefficients only. Also we will work only with continuous piecewise affine maps.

Let $\sigma: \mathbb{R} \rightarrow \mathbb{R}$ be the piecewise affine map such that $\sigma(x)=x$ for $x \in[-1,1], \sigma(x)=1$ for $x \geq 1$, and $\sigma(x)=-1$ for $x \leq-1$. A saturated linear map is a piecewise affine function $f: \mathbb{R}^{p} \rightarrow \mathbb{R}^{q}$ of the form $f(x)=\sigma(A x)$. Here $\sigma$ is applied componentwise. Naturally, we say that $A$ is the matrix associated to $f$. 
Theorem 3, our undecidability result for saturated linear maps, is obtained by a reduction from the problem of Theorem 2: deciding whether the entropy of a continuous piecewise affine map is equal to zero. In order to obtain this reduction, we find it convenient to prove Theorem 2 for a restricted class of continuous piecewise affine maps: the so-called $\sigma_{0}^{*}$ functions. We recall from [I] that a $\sigma_{0}^{*}$-function of depth $d$ is a composition of $d$ saturated linear functions. Note that $f: \mathbb{R}^{p} \rightarrow \mathbb{R}^{q}$ is a $\sigma_{0}^{*}$-function iff each of its $q$ components is a $\sigma_{0}^{*}$-function (for the if part, note that we may assume that the $q$ components have the same depth since $\sigma(g)=g$ for any $\sigma_{0}^{*}$-function $\left.g: \mathbb{R}^{p} \rightarrow \mathbb{R}\right)$.

\section{Undecidable Properties}

Our undecidability results are based on reductions from the "hyperplane problem" [i]].

Theorem 1 (Theorem 7 of [1]]) The following decision problem is undecidable.

- Instance: three $\sigma_{0}^{*}$-functions $f, g, Z: \mathbb{R}^{3} \rightarrow \mathbb{R}$.

- Question: does there exist a trajectory of the system $\left(x_{t+1}, y_{t+1}\right)=$ $\left(f\left(x_{t}, y_{t}, 1\right), g\left(x_{t}, y_{t}, 1\right)\right)$ which satisfies $Z\left(x_{t}, y_{t}, 1\right)=0$ for all $t$ ?

Let us recall some constructions from [1]. Sel $: \mathbb{R}^{2} \rightarrow \mathbb{R}$ is a $\sigma_{0}^{*}$-function which is zero in a neighbourhood of 0 , and satisfies

1. $\operatorname{Sel}(1, e)=e$

2. $\operatorname{Sel}(0, e)=0$

for all $e \in[-1,1]$.

In the same paper we constructed a $\sigma_{0}^{*}$-function $S t a b: \mathbb{R}^{2} \rightarrow \mathbb{R}$ which is zero in a neighbourhood of 0 , and satisfies the following property. For all $z_{0} \in \mathbb{R}$ and for all functions $e: \mathbb{N} \rightarrow \mathbb{R}$, the sequence $z_{t+1}=\operatorname{Stab}\left(z_{t}, e_{t}\right)$ falls into one of the following three mutually exclusive cases:

1. The sequence $z_{t}, t \geq 1$ is constant, always equal to 1 . This case happens only when $\sigma\left(z_{0}\right)=1$ and when $e_{t}=0$ for all $t$.

2 . The sequence $z_{t}, t \geq 1$ is constant, always equal to -1 . This case happens only when $\sigma\left(z_{0}\right)=-1$ and when $e_{t}=0$ for all $t$.

3. The sequence $z_{t}$ is eventually null: there exists $t_{0}$ with $z_{t}=0$ for all $t \geq t_{0}$.

It is shown in [U] that one may take $\operatorname{Sel}(z, e)=\sigma(2 h(3 z / 4+e / 4)-h(z))$ where $h$ denotes the function $h(x)=\sigma(2 \sigma(x)-\sigma(2 x))$. For Stab one may take $\operatorname{Stab}(z, e)=h(\sigma(\sigma(z))-$ $A b s(z, e) / 2)$, where $A b s(z, e)=\sigma(\sigma(e-z)-\sigma(e+z)+2 \sigma(z))$.

Theorem 2 It is undecidable whether the topological entropy of a given $\sigma_{0}^{*}$-function $F$ : $[-1,1]^{4} \rightarrow[-1,1]^{4}$ is equal to zero.

This problem remains unsolvable even if we are promised that $F$ satisfies the following condition: if $h(F)=0$ then $F$ is nilpotent.

Proof. We reduce the problem of Theorem 1 to this problem. let $f, g, Z: \mathbb{R}^{3} \rightarrow \mathbb{R}$ be three $\sigma_{0}^{*}$-functions. Consider the following four-dimensional system $X_{t+1}=F\left(X_{t}\right)$ :

$$
\begin{aligned}
& w_{t+1}=\operatorname{Sel}\left(z_{t}, \Psi\left(w_{t}, z_{t}\right)\right) \\
& x_{t+1}=\operatorname{Sel}\left(z_{t}, f\left(x_{t}, y_{t}, z_{t}\right)\right) \\
& y_{t+1}=\operatorname{Sel}\left(z_{t}, g\left(x_{t}, y_{t}, z_{t}\right)\right) \\
& z_{t+1}=\operatorname{Stab}\left(z_{t}, Z\left(x_{t}, y_{t}, z_{t}\right)\right)
\end{aligned}
$$

Here $\psi$ is the $\sigma_{0}^{*}$-function $\psi(w, z)=\sigma((\sigma(4 w-z)+\sigma(3 z-4 w)) / 2)$. For $w \in[0,1]$, note that $\psi(w, 1)$ is equal to $\phi(w)$, the tent map from section 2.1. Note also that $F$ is a $\sigma_{0}^{*}$-function since its four components are $\sigma_{0}^{*}$-functions.

We claim that the four following properties are equivalent. 
(i) No trajectory of the system $\left(x_{t+1}, y_{t+1}\right)=\left(f\left(x_{t}, y_{t}, 1\right), g\left(x_{t}, y_{t}, 1\right)\right)$ satisfies $Z\left(x_{t}, y_{t}, 1\right)=0$ for all $t$.

(ii) $F$ is mortal.

(iii) $F$ is nilpotent.

(iv) The topological entropy of $F$ is equal to zero.

Theorem 2 then follows immediately from Theorem 1 and the equivalence of (i), (iii) and (iv).

Assume first that (i) holds. To show that $F$ is mortal, we only need to consider trajectories with $z_{1} \in[0,1]$ since $F$ is an odd function. If $z_{t}$ is always constant and equal to 1 then by construction of Sel and Stab, $\left(x_{t}, y_{t}\right)$ is a trajectory of the system $\left(x_{t+1}, y_{t+1}\right)=\left(f\left(x_{t}, y_{t}, 1\right), g\left(x_{t}, y_{t}, 1\right)\right)$ which satisfies $Z\left(x_{t}, y_{t}, 1\right)=0$ for all $t$. This is impossible by hypothesis. By construction of Stab, $z_{t}$ must therefore be eventually null. By construction of $S e l$, this implies that $w_{t}, x_{t}$ and $y_{t}$ are also eventually null: the system's trajectory is indeed mortal.

Since $S e l$ and Stab are zero in a neighbourhood of zero, the same is true of $F$ : there exists an open neighbourhood $U$ of 0 such that $F(U)=\{0\}$. The implication from mortality to nilpotency then follows by compactness: if $F$ is mortal then $[-1,1]^{4}=$ $\bigcup_{n \geq 0} F^{-n}(U)$. Hence there is a finite cover of the form $[-1,1]^{4}=\bigcup_{n=0}^{N} F^{-n}(U)$, which implies $F^{N+1}\left([-1,1]^{4}\right)=\{0\}$.

As pointed out in section 2.1, (iii) implies (iv). Finally, in order to show that (iv) implies (i) we show that $\neg$ (i) implies $\neg$ (iv). Consider a trajectory of the system $\left(x_{t+1}, y_{t+1}\right)=$ $\left(f\left(x_{t}, y_{t}, 1\right), g\left(x_{t}, y_{t}, 1\right)\right)$ which satisfies $Z\left(x_{t}, y_{t}, 1\right)=0$ for all $t$. For any initial value $w_{0} \in$ $[0,1]$ we obtain a trajectory of $F$ which satisfies $w_{t+1}=\phi\left(w_{t}\right)$ and $z_{t}=1$ for all $t$. This implies that $s_{t}(\varepsilon, F) \geq s_{t}(\varepsilon, \phi)$ for all $t, \varepsilon>0$, so that $h(F) \geq h(\phi)>0$.

Note that the piecewise-affine function $F$ constructed in this proof either satisfies $h(F)=0$ or $h(F) \geq \ln 2$. This implies that for $\varepsilon<\ln 2$ no algorithm can approximate the topological entropy of a piecewise-affine with an absolute error bounded by $\varepsilon$. A variation on this construction would show just as in [2] that the topological entropy cannot be approximated up to $\varepsilon$, no matter how large $\varepsilon$ is.

Theorem 3 It is undecidable whether the topological entropy of a given saturated linear function is equal to zero.

This problem remains unsolvable even if we are promised that the saturated linear function is nilpotent if its topological entropy is equal to zero.

Proof. We reduce the problem of Theorem 2 to this problem. Let $F:[-1,1]^{4} \rightarrow[-1,1]^{4}$ be a $\sigma_{0}^{*}$-function. To this function we can associate a saturated linear function $F^{\prime}$ as in [I]. This is done as follows. Assume that $F$ is of the form $f_{k} \circ f_{k-1} \circ \ldots \circ f_{1}$ for some $\sigma_{0}$-functions $f_{j}(x)=\sigma\left(A_{j} x\right)$, where $f_{j}: \mathbb{R}^{d_{j-1}} \rightarrow \mathbb{R}^{d_{j}}$ with $d_{0}, d_{1}, \ldots, d_{k} \in \mathbb{N}$, and $d_{0}=d_{k}=4$.

Let $d=d_{0}+d_{1}+\cdots+d_{k}$, and consider the saturated linear function $F^{\prime}: \mathbb{R}^{d} \rightarrow \mathbb{R}^{d}$ defined by $F^{\prime}(x)=\sigma(A x)$ where

$$
A=\left(\begin{array}{lllll}
0 & 0 & \ldots & 0 & A_{k} \\
A_{1} & 0 & \ldots & 0 & 0 \\
0 & A_{2} & \ldots & 0 & 0 \\
\vdots & \vdots & & 0 & 0 \\
0 & 0 & \ldots & A_{k-1} & 0
\end{array}\right)
$$

As pointed out in [U], it is clear that if $F^{m} \equiv 0$ for some integer $m$ then $F^{\prime k m} \equiv 0$, so that $h\left(F^{\prime}\right)=0$. Conversely, consider a trajectory $X_{t+1}=F\left(X_{t}\right)$ of $F$, and a trajectory $X_{t+1}^{\prime}=$ $F^{\prime}\left(X_{t}^{\prime}\right)$ such that the first $d_{0}$ components of $X_{0}^{\prime}$ are equal to $X_{0}$. The first $d_{0}$ components 
of $X_{k t}^{\prime}$ are then equal to $X_{t}$ for all $t \geq 0$. This implies that $s_{t k}\left(\varepsilon, F^{\prime}\right) \geq s_{t}(\varepsilon, F)$ so that $h\left(F^{\prime}\right) \geq h(F) / k$.

We can now conclude. Assume that we are promised that $F$ is nilpotent if $h(F)=0$. We have shown that:

(i) if $h(F)=0$ then $h\left(F^{\prime}\right)=0$ and $F^{\prime}$ is nilpotent.

(ii) if $h(F)>0$ then $h\left(F^{\prime}\right)>0$.

The problem of Theorem $[$ is therefore undecidable.

\section{Nilpotent Systems in Fixed Dimension}

It was pointed out in [I] that our undecidability results have "purely mathematical" consequences. For instance, there is no recursive function $s(n)$ such that all saturated linear maps $f: \mathbb{R}^{n} \rightarrow \mathbb{R}^{n}$ satisfy $f^{s(n)}=0$. Note in contrast that the bound $s(n)=n$ is valid for nilpotent linear maps. The existence of such a bound for saturated linear maps would imply the decidability of their nilpotency problem, but this problem is undecidable [I]. This observation leaves us with the following alternative:

(i) either there is for all $n$ a finite bound $s(n)$ such that all saturated linear maps $f: \mathbb{R}^{n} \rightarrow$ $\mathbb{R}^{n}$ satisfy $f^{s(n)}=0$ (and in this case $s$ must be a very fast growing function).

(ii) or for some integer $n$ no finite bound exists.

We now show that the second branch of the alternative is the correct one.

Proposition 1 There exists an integer $n$ such that for any integer $k$ there exists a nilpotent saturated linear map $f: \mathbb{R}^{n} \rightarrow \mathbb{R}^{n}$ with $f^{k} \neq 0$.

This follows from Lemma 1 below (note again the contrast with the case of linear maps).

Lemma $1 \mathrm{Let} \mathrm{Nil}_{n}$ be the set of matrices of nilpotent saturated linear maps in dimension $n$. There exists an integer $n$ such that $N_{i} l_{n}$ is not a closed subset of $\mathbb{R}^{n^{2}}$.

Proof. Consider the one dimensional system $z_{t+1}=F_{\varepsilon}\left(z_{t}\right)$, where $F_{\varepsilon}$ is the $\sigma_{0}^{*}$-function $z_{t} \mapsto \operatorname{Stab}\left(z_{t}, \varepsilon z_{t}\right)$. Here $\varepsilon$ is a real parameter. For $\varepsilon=0$ the system is not nilpotent since 1 is a fixed point. For $\varepsilon \neq 0$ the system is mortal by the third property of Stab. Since $F_{\varepsilon}$ is identically zero in a neighborhood of zero, this implies by compactness (as in the proof of Theorem (3) that $F_{\varepsilon}$ is in fact nilpotent.

Consider now as in (11) the matrix $A_{\varepsilon}$ of the saturated linear map $F_{\varepsilon}^{\prime}$ which is associated to $F_{\varepsilon}$. This $\sigma_{0}^{*}$-function is nilpotent if and only if $F_{\varepsilon}^{\prime}$ is nilpotent, and as a consequence $A_{\varepsilon}$ is in $N i l_{n}$ if and only if $\varepsilon \neq 0$.

Proof of Theorem प. Let $N i l_{n, k}$ be the set of matrices of saturated linear maps $f: \mathbb{R}^{n} \rightarrow \mathbb{R}^{n}$ such that $f^{k}=0$. By continuity each $N i l_{n, k}$ is a closed subset of $\mathbb{R}^{n^{2}}$, but we have just seen that $N i l=\cup_{k \geq 1} N i l_{n, k}$ is not closed. We conclude that $N i l_{n} \backslash N i l_{n, k} \neq \emptyset$ for all $k$.

It follows from the explicit formula for Stab given before Theorem 2 that one may take $n=9$ in Lemma 1 and Proposition 1 . 


\section{References}

[1] V. Blondel, O. Bournez, P. Koiran, and J. Tsitsiklis. The stability of saturated linear dynamical systems is undecidable. Journal of Computer and System Sciences, 62:442-462, 2001. short version presented at STACS 2000.

[2] L. Hurd, J. Kari, and K. Culik. The topological entropy of cellular automata is uncomputable. Ergodic Theory and Dynamical Systems, 12:255-265, 1992.

[3] J. Kari. The nilpotency problem of one-dimensional cellular automata. SIAM Journal on Computing, 21:571-586, 1992.

[4] Ker-I Ko. Complexity Theory of Real Functions. Birkhäuser, 1991.

[5] R. Mañé. Ergodic Theory and Differentiable Dynamics. Springer-Verlag, New York, 1987.

[6] M. Misiurewicz and W. Szlenc. Entropy of piecewise monotone mappings. Studia Mathematica, 67:45-63, 1980. preliminary version in Astérisque 50.

[7] E. D. Sontag. From linear to nonlinear: some complexity comparisons. In Proc. IEEE Conference on Decision and Control, pages 2916-2920, 1995.

[8] P. Walters. An Introduction to Ergodic Theory. Graduate Texts in Mathematics. Springer, 1982.

[9] K. Weihrauch. Computability. EATCS Monographs on Theoretical Computer Science. Springer, 1987.

[10] X. Zheng and K. Weihrauch. The arithmetical hierarchy of real numbers. In Proc. MFCS 1999, volume 1672 of Lecture Notes in Computer Science, pages 23-33. Springer-Verlag, 1999. 